\title{
Erratum: Exceptional points in anisotropic planar microcavities [Phys. Rev. A 95, 023836 (2017)]
}

\author{
Steffen Richter, Tom Michalsky, Chris Sturm, Bernd Rosenow, \\ Marius Grundmann, and Rüdiger Schmidt-Grund \\ (Received 20 October 2017; published 9 November 2017)
}

DOI: 10.1103/PhysRevA.96.059902

An error in the computational code lead to incorrect $\tilde{P}_{1}$ and $\tilde{P}_{2}$ components in Figs. 2, 3, and 6. In particular, the sign of $2 \varphi$ for the rotation of the linear polarization as correctly given by Eqs. (A9) and (A10) was incorrectly implemented. For forward-traveling modes, this rotation of the polarization pseudospin vector has to be performed in the same direction as the coordinate rotation due to $\varphi$ for the dielectric tensor according to Eqs. (A8) and (A7). As stated in the article, with the definition of Eq. (A7) this is true for the upper signs of the \pm and $\mp$ in Eqs. (A9) and (A10). This was incorrect in the earlier implementation.

The correction has no consequences on any of the discussed effects, i.e., the structure of complex mode energies and the properties of the exceptional points (EPs). The EPs remain circularly polarized and still establish vortices for the linear polarization components $\left(\tilde{P}_{1}, \tilde{P}_{2}\right)^{\mathrm{T}}$ with winding number +1 when encircling a pair of EPs (winding number $+1 / 2$ for a single EP). However, the $\tilde{P}_{1}$ and $\tilde{P}_{2}$ components are modified. One mode is mostly polarized parallel, while the other one is mostly perpendicular to the optic axis of the uniaxial cavity medium. The seeming vortex of $\left(\tilde{P}_{1}, \tilde{P}_{2}\right)^{\mathrm{T}}$ around the origin $\vec{k}_{\|}=\overrightarrow{0}$ was artificial and has disappeared now. In the following, we show the corrected plots which replace Figs. 2, 3, and 6 of the original article, respectively.

We would like to sincerely thank Heinrich-Gregor Zirnstein for his help in tracing back the origin of the error, including code review.
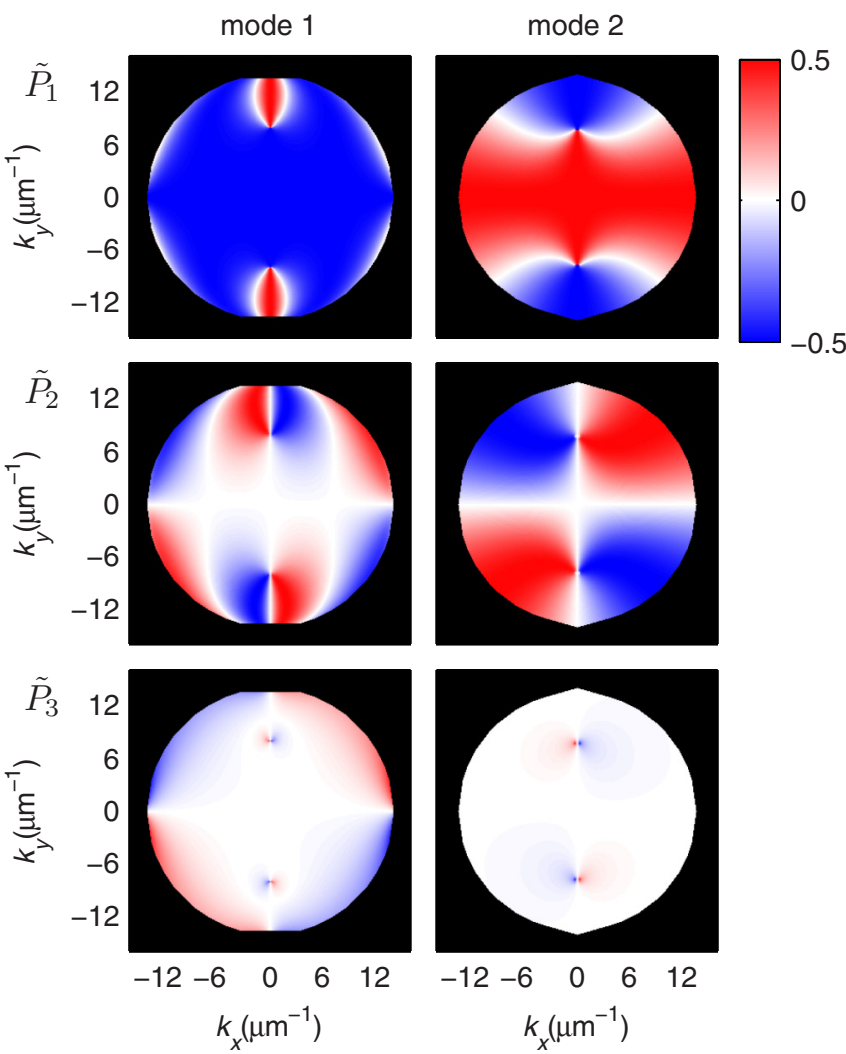

FIG. 2. Mode polarization in terms of the pseudospin. Replacement for Fig. 2 of the original article. Changes occur only in $\tilde{P}_{1}$ and $\tilde{P}_{2}$. 


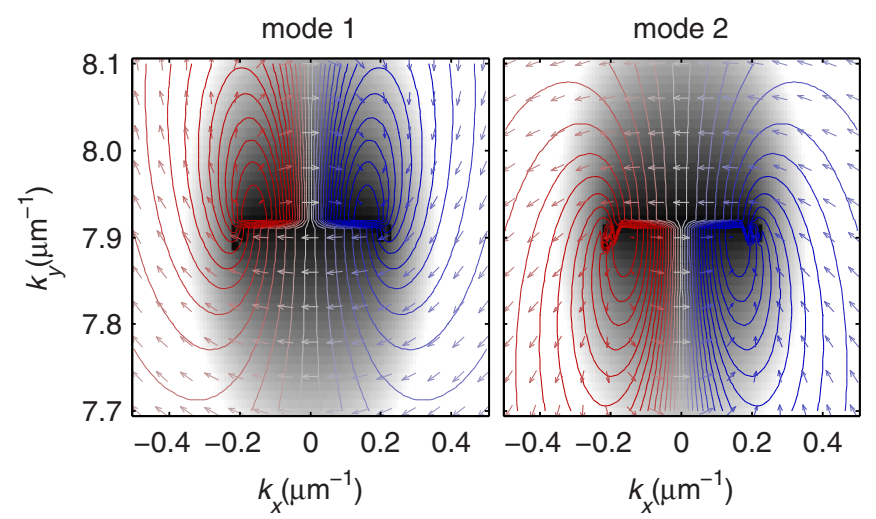

FIG. 3. Detailed view of the polarization of the two modes in the momentum space range around a pair of EPs. Replacement for Fig. 3 of the original article. Changes affect only the direction of the arrows. The vortex characteristics are conserved.

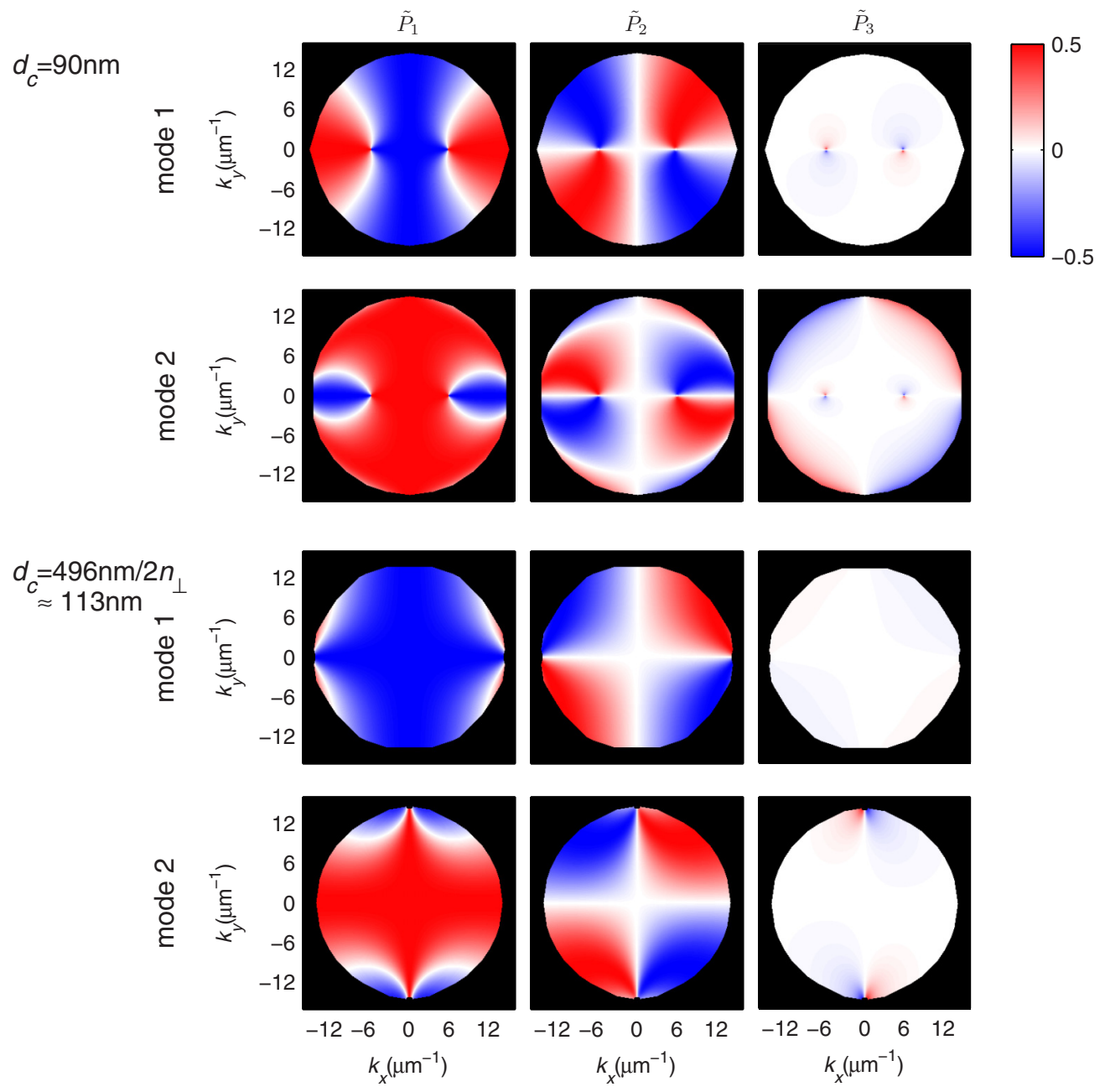

FIG. 6. Further mode polarization patterns in terms of the pseudospin. Replacement for Fig. 6 in the appendix of the original article. 